\title{
Deprojecting spiral galaxies using Fourier analysis. Application to the Frei sample ${ }^{\star}$
}

\author{
C. Barberà ${ }^{1}$, E. Athanassoula ${ }^{2}$, and C. García-Gómez ${ }^{1}$ \\ 1 D.E.I.M., Campus Sescelades, Avd. dels Països Catalans 26, 43007 Tarragona, Spain \\ 2 Observatoire de Marseille, 2 Place Le Verier, 13248 Marseille cedex 04, France
}

Received 12 August 2003 / Accepted 30 September 2003

\begin{abstract}
We present two methods that can be used to deproject spirals, based on Fourier analysis of their images, and discuss their potential and restrictions. Our methods perform particularly well for galaxies more inclined than $50^{\circ}$ or for non-barred galaxies more inclined than $35^{\circ}$. They are fast and straightforward to use, and thus ideal for large samples of galaxies. Moreover, they are very robust for low resolutions and thus are appropriate for samples of cosmological interest. The relevant software is available from us upon request. We use these methods to determine the values of the position and inclination angles for a sample of 79 spiral galaxies contained in the Frei et al. (1996) sample. We compare our results with the values found in the literature, based on other methods. We find statistically very good agreement
\end{abstract}

Key words. galaxies: structure - galaxies: spiral

\section{Introduction}

Disc galaxies are observed projected on the sky. Yet for many purposes one needs to be able to deproject them. Thus in morphological studies one needs information on different sizes and their ratios, e.g. to obtain the shape of rings, the axial ratio and strength of bars, or the shape (diamond-like, elliptical, or rectangular-like) of bar isophotes. Similarly in photometrical studies one may need cuts along particular directions, like the bar major or minor axis, or one may need to compare arm with inter-arm regions located at the same distance from the center, or one may need to correct for extinction. For studies involving the Tully-Fisher relation one needs to know the inclination of the galaxy in order to obtain its velocity. Our own interest in deprojection angles stems from the fact that we started a quantitative study of the properties of spiral structure in near-by disc galaxies, and for this we first need to deproject all our images. Indeed the list of all studies for which it is necessary to know the spatial orientation of the galaxy is too long to include here.

We will study the spiral structure in disc galaxies decomposing each image by means of bidimensional Fourier transforms. The first step is to deproject the galaxy image. It is thus necessary to determine the two deprojection angles, namely the position angle (hereafter PA) and the inclination angle (hereafter IA). The PA is the angle between the line of nodes of the projected image and the north, measured towards the east,

Send offprint requests to: $\mathrm{C}$. Barberà,

e-mail: cbarbera@etse.urv.es

* Table 7 is only available in electronic form at the CDS via anonymous ftp to cdsarc.u-strasbg.fr $(130.79 .128 .5)$ or via http://cdsweb.u-strasbg.fr/cgi-bin/qcat?J/A+A/415/849 while the IA is the angle between the perpendicular to the plane of the galaxy and the line of sight. Several methods have been proposed so far to obtain these angles, the most commonly used ones being based on photometry or kinematics. A standard way is to fit ellipses to the outer isophotes. The axis ratio of the outer ellipses is a measure of the IA, while the direction of the major axis gives the PA. Another procedure is based on the gas kinematics within the disc, using two dimensional velocity fields and assuming that the emission comes from material in a thin disk in circular motion around the center. The selected PA and IA angles are those that minimize the departures from such a flow.

These are not the only methods used in the literature. Danver (1942) used a special display table to rotate the galaxy images until they were circular. Grøsbol (1985) applied a one dimensional Fourier transform to the intensity distribution in the outer parts of galaxy disks and adopted the deprojection angles that minimized the bisymmetric $(m=2)$ Fourier coefficient. Comte et al. (1979) used the distribution of HII regions in the $\ln (r)-\theta$ plane to fit straight lines to the arms, under the hypothesis that they are well described by logarithmic spirals. Two dimensional Fourier analysis has also been used to determine the deprojection angles. Considère \& Athanassoula (1982) used the HII region distribution, selecting the angles that maximized the signal-to-noise ratio in the $m=2$ component, and Considère \& Athanassoula (1988), applied the same criterion to galaxy images. Iye et al. (1982) used an image of NGC 4254 and selected the angles that maximized the axisymmetric component. Finally García-Gómez \& Athanassoula (1991) and García-Gómez et al. (2002) also used 
HII region distributions and maximized the axisymmetric components These authors also used a second method selecting the deprojection angles that made the HII region distribution most uniform.

All methods used for the deprojection of disk galaxies suffer from some kind of systematic errors. They will all work properly in the case of perfectly axisymmetric thin disks in circular motion about their centers, which is never the case for real galaxies. Thus, when we fit ellipses to the outer isophotes, the presence of strong arms can bias the results. This bias is known as Stocke's effect (Stocke 1955). On the other hand, Athanassoula \& Misiriotis (2002) show that, even for very strong bars, the isophotes in the outermost part of the disc are sufficiently circular to be used for deprojection, provided that there is no spiral structure and one can go sufficiently far out from the center of the disc. The reason for this difference is that spiral arms extend far out in the disk, while bars are confined to the inner parts. Kinematical methods can be biased by warps in the outer parts, or by non-circular motions in the central parts due to bars or other perturbations.

The two deprojection methods, which we will introduce here, are based on the Fourier analysis of galaxy images. In Sects. 2 and 3 we introduce the sample and the methods, respectively. In Sect. 4 we assess our two methods and apply them to our sample. In Sect. 5 we compare the values obtained by the different methods, i.e. our two methods and those previously used in the literature. This will allow a quantitative comparison of the performance of the different methods. In Sect. 6, we adopt the values of the PA and IA that we will use in our quantitative studies of the spiral structure in the sample of galaxies. We summarize in Sect. 7.

\section{The sample}

Frei et al. (1996) presented a catalog of images of 113 galaxies in different pass-bands. All galaxies are nearby, bright and well resolved and were selected to span all the Hubble classification classes. This catalog is thus ideal for statistical studies.

As we are interested in the spiral structure of disc galaxies, we selected from the Frei sample all the galaxies with Hubble types between 2 and 7. We included also the galaxies NGC 4340, NGC 4866 and NGC 5701 with Hubble types -1 and 0 and the galaxy NGC 4178 with Hubble type 8, as their disks have a regular shape and they are not too inclined. For the same reason, we discard nearly edge-on galaxies, in which structure is not visible.

We are left with a sample of 79 galaxies, the general properties of which are listed in Table 1. Column 1 gives the galaxy name, Col. 2 the Hubble type and Col. 3 this type as coded in the RC3 catalog (de Vaucouleurs 1991). Column 4 gives the arm class as defined in Elmegreen \& Elmegreen (1987). Finally, Col. 5 gives the filters used when observing each of the galaxy images. Figure 1 shows the distribution of galaxy types for our sample. We note that Hubble types are not uniformly sampled, since types Sbc to Scd are clearly over-represented. Figure 2 shows the distribution of arm classes in the galaxy sample. Again the different arm classes are not uniformly
Table 1. Properties of the galaxies in the sample.

\begin{tabular}{|c|c|c|c|c|}
\hline Name & $\overline{\mathrm{T}}$ & $\bar{S}$ & $\overline{\mathrm{AC}}$ & Filters \\
\hline NGC 2403 & .SXS6.. & 6.0 & 4 & $g, i, r$ \\
\hline NGC 2541 & .SAS6.. & 6.0 & 1 & $g, i, r$ \\
\hline NGC 2683 & .SAT3.. & 3.0 & - & $B_{J}, R$ \\
\hline NGC 2715 & .SXT5.. & 5.0 & - & $B_{J}, R$ \\
\hline NGC 2775 & .SAR2.. & 2.0 & 3 & $B_{J}, R$ \\
\hline NGC 2903 & .SXT4.. & 4.0 & 4 & $g, i, r$ \\
\hline NGC 2976 & .SA.5P. & 5.0 & 3 & $B_{J}, R$ \\
\hline NGC 2985 & PSAT2.. & 2.0 & 3 & $B_{J}, R$ \\
\hline NGC 3031 & .SAS2.. & 2.0 & 12 & $g, i, r$ \\
\hline NGC 3079 & SBS5. & 7.0 & - & $B_{J}, R$ \\
\hline NGC 3147 & .SAT4.. & 4.0 & 3 & $B_{J}, R$ \\
\hline NGC 3184 & .SXT6.. & 6.0 & 9 & $B_{J}, R$ \\
\hline NGC 3198 & .SBT5.. & 5.0 & - & $g, i, r$ \\
\hline NGC 3319 & .SBT6.. & 6.0 & 5 & $g, i, r$ \\
\hline NGC 3344 & RSXR4.. & 4.0 & 9 & $B_{J}, R$ \\
\hline NGC 3351 & .SBR3.. & 3.0 & 6 & $B_{J}, R$ \\
\hline NGC 3368 & .SXT2.. & 2.0 & 8 & $B_{J}, R$ \\
\hline NGC 3486 & .SXR5.. & 5.0 & 9 & $B_{J}, R$ \\
\hline NGC 3596 & .SXT5.. & 5.0 & 5 & $B_{J}, R$ \\
\hline NGC 3623 & .SXT1.. & 1.0 & - & $B_{J}, R$ \\
\hline NGC 3631 & .SAS5.. & 5.0 & 9 & $B_{J}, R$ \\
\hline NGC 3672 & .SAS5.. & 5.0 & 2 & $B_{J}, R$ \\
\hline NGC 3675 & .SAS3.. & 3.0 & 3 & $B_{J}, R$ \\
\hline NGC 3726 & .SXR5.. & 5.0 & 5 & $B_{J}, R$ \\
\hline NGC 3810 & .SAT5.. & 5.0 & 2 & $B_{J}, R$ \\
\hline NGC 3877 & .SAS5* & 5.0 & - & $B_{J}, R$ \\
\hline NGC 3893 & .SXT5* & 5.0 & 12 & $B_{J}, R$ \\
\hline NGC 3938 & .SAS5.. & 5.0 & 9 & $B_{J}, R$ \\
\hline NGC 3953 & .SBR4.. & 4.0 & 9 & $B_{J}, R$ \\
\hline NGC 4030 & .SAS4.. & 4.0 & 9 & $B_{J}, R$ \\
\hline NGC 4088 & .SXT4.. & 4.0 & - & $B_{J}, R$ \\
\hline NGC 4123 & .SBR5.. & 5.0 & 9 & $B_{J}, R$ \\
\hline NGC 4136 & .SXR5.. & 5.0 & 9 & $B_{J}, R$ \\
\hline NGC 4144 & .SXS6\$. & 6.0 & - & $B_{J}, R$ \\
\hline NGC 4157 &. $\mathrm{SXS} 3 \$$ & 3.0 & - & $B_{J}, R$ \\
\hline NGC 4178 & .SBT8.. & 8.0 & - & $g, i, r$ \\
\hline NGC 4189 & .SXT6\$. & 6.0 & 2 & $g, i, r$ \\
\hline NGC 4192 & $. S X S 2 .$. & 2.0 & - & $g, i, r$ \\
\hline NGC 4216 &. $\mathrm{SXS} 3 *$ & 3.0 & - & $g, i, r$ \\
\hline NGC 4254 & .SAS5.. & 5.0 & 9 & $g, i, r$ \\
\hline NGC 4258 & $. S X S 4 .$. & 4.0 & - & $g, i, r$ \\
\hline
\end{tabular}

sampled. In the present sample, arm class 9 is much better represented than the other arm classes.

\section{Deprojection methods}

In this paper we introduce two methods, based on the Fourier transforms and which are closely linked to the two methods used by García-Gómez et al. (2002) for HII region distributions. Let $I(u, \theta)$ be the image of the galaxy written in polar 
Table 1. continued.

\begin{tabular}{|c|c|c|c|c|}
\hline Name & $\mathrm{T}$ & $S$ & $\mathrm{AC}$ & Filters \\
\hline NGC 4303 & .SXT4.. & 4.0 & 9 & $g, i, r$ \\
\hline NGC 4321 &. SXS4.. & 4.0 & 12 & $g, i, r$ \\
\hline NGC 4340 & .LBR+.. & -1.0 & - & $B_{J}, R$ \\
\hline NGC 4394 & RSBR3.. & 3.0 & 6 & $B_{J}, R$ \\
\hline NGC 4414 & .SAT5\$. & 5.0 & 3 & $g, i, r$ \\
\hline NGC 4450 & .SAS2.. & 4.0 & 12 & $B_{J}, R$ \\
\hline NGC 4487 & .SXT6.. & 6.0 & 5 & $B_{J}, R$ \\
\hline NGC 4498 & $. S X S 7 .$. & 6.5 & 4 & $g, i, r$ \\
\hline NGC 4501 & .SAT3.. & 3.0 & 9 & $g, i, r$ \\
\hline NGC 4527 &. SXS4.. & 4.0 & - & $g, i, r$ \\
\hline NGC 4535 & .SXS5.. & 5.0 & 9 & $g, i, r$ \\
\hline NGC 4548 & .SBT3.. & 3.0 & 5 & $g, i, r$ \\
\hline NGC 4559 & .SXT6.. & 6.0 & - & $g, i, r$ \\
\hline NGC 4569 & .SXT2.. & 2.0 & - & $g, i, r$ \\
\hline NGC 4571 & .SAR7.. & 6.5 & - & $g, i, r$ \\
\hline NGC 4579 & .SXT3.. & 3.0 & 9 & $g, i, r$ \\
\hline NGC 4593 & RSBR3.. & 3.0 & 5 & $B_{J}, R$ \\
\hline NGC 4651 & .SAT5.. & 5.0 & 9 & $g, i, r$ \\
\hline NGC 4654 & .SXT6.. & 6.0 & 4 & $g, i, r$ \\
\hline NGC 4689 & .SAT4.. & 4.0 & 3 & $g, i, r$ \\
\hline NGC 4725 & .SXR2P. & 2.0 & 6 & $g, i, r$ \\
\hline NGC 4826 & RSAT2.. & 2.0 & 6 & $g, i, r$ \\
\hline NGC 4866 & $. \mathrm{LAR}+* /$ & -1.0 & - & $B_{J}, R$ \\
\hline NGC 5005 &. SXT4.. & 4.0 & 3 & $B_{J}, R$ \\
\hline NGC 5033 & .SAS5.. & 5.0 & 9 & $g, i, r$ \\
\hline NGC 5055 &. SXT4.. & 4.0 & 3 & $g, i, r$ \\
\hline NGC 5248 & .SXT4.. & 4.0 & 12 & $B_{J}, R$ \\
\hline NGC 5334 & .SBT5* & 5.0 & 2 & $g, i, r$ \\
\hline NGC 5364 & .SAS6.. & 4.0 & 9 & $B_{J}, R$ \\
\hline NGC 5371 &. SXT4.. & 4.0 & 9 & $B_{J}, R$ \\
\hline NGC 5585 & $. S X S 7 .$. & 7.0 & 1 & $B_{J}, R$ \\
\hline NGC 5669 & .SXT6.. & 6.0 & 5 & $B_{J}, R$ \\
\hline NGC 5701 & RSBT0.. & 0.0 & - & $B_{J}, R$ \\
\hline NGC 5792 & .SBT3.. & 3.0 & - & $B_{J}, R$ \\
\hline NGC 5850 & .SBR3.. & 3.0 & 8 & $B_{J}, R$ \\
\hline NGC 5985 & .SXR3.. & 3.0 & 9 & $B_{J}, R$ \\
\hline NGC 6015 & .SAS6.. & 6.0 & - & $B_{J}, R$ \\
\hline NGC 6118 & .SAS6.. & 6.0 & - & $B_{J}, R$ \\
\hline NGC 6384 & .SXR4.. & 4.0 & 9 & $B_{J}, R$ \\
\hline NGC 6503 & .SAS6.. & 6.0 & - & $B_{J}, R$ \\
\hline
\end{tabular}

coordinates $(r, \theta)$, and $u=\ln (r)$. We define the Fourier transform of this image as:

$A(p, m)=\int_{u_{\min }}^{u_{\max }} \int_{0}^{2 \pi} I(u, \theta) \exp [i(p u+m \theta)] \mathrm{d} \theta \mathrm{d} u$.

In this equation, $p$ corresponds to the radial frequency and $m$ to the azimuthal frequency. Thus the $m=1$ values correspond to one-armed components, the $m=2$ values to twoarmed components and so on. The values of $u_{\min }=\ln \left(r_{\min }\right)$ and $u_{\max }=\ln \left(r_{\max }\right)$ are set by the inner and outer radius of the image, or of the part that we will analyze.

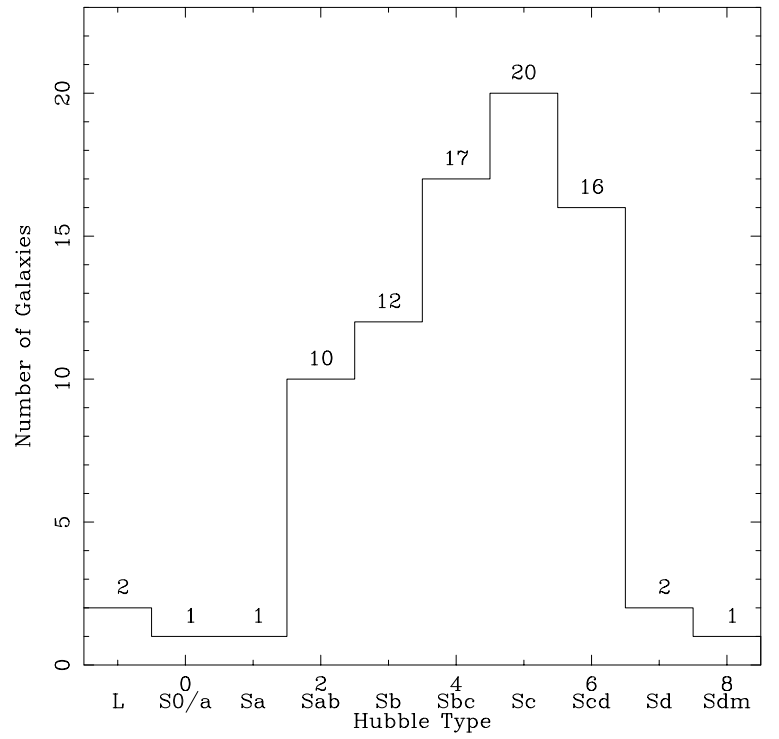

Fig. 1. Galaxy distribution as a function of galaxy type.

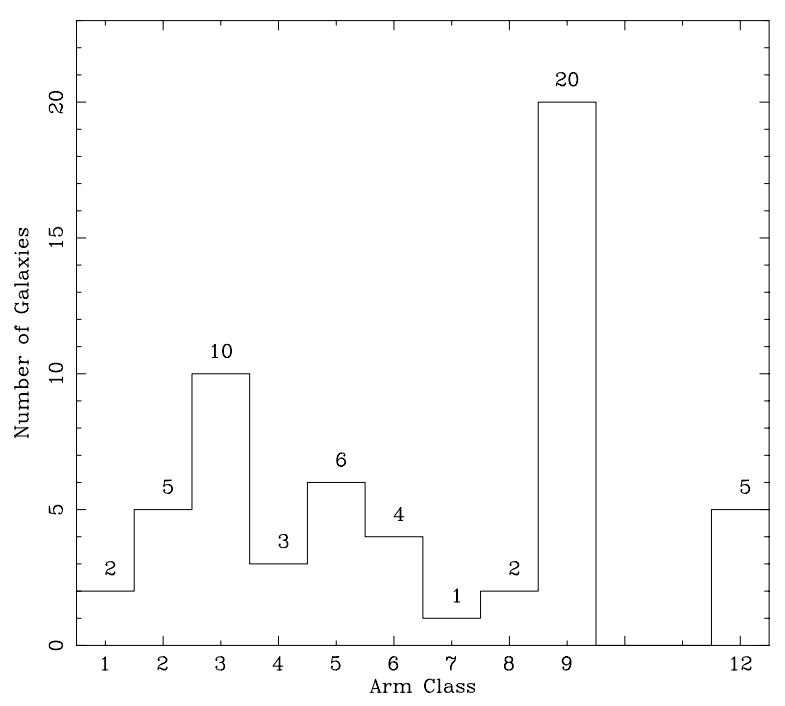

Fig. 2. Galaxy distribution as a function of arm class.

Fixing the value of $m$, we can calculate the power associated to this component simply as:

$P_{m}=|A(p, m)|=\left|\int_{-p_{\max }}^{p_{\max }} A(p, m) \mathrm{d} p\right|$.

The value of $p_{\max }$ is related to the resolution in Fourier space through

$p_{\max }=\frac{1}{2 \Delta u}=\frac{N-1}{2\left(u_{\max }-u_{\min }\right)}$,

where $N$ is the number of points used in the Fourier transform in the radial dimension, usually $N=256$ or $N=512$.

In our first method we try to minimize the effect of the spiral structure by minimizing the ratio:

BAG1 $=\frac{P_{1}+P_{2}+\cdots+P_{6}}{P_{0}+P_{1}+\cdots+P_{6}}$

This is equivalent to maximizing the contribution of the axisymmetric component. Since a badly deprojected galaxy will 
look oval, and thus contribute to the $m=2$ component as a bar, for our second method we simply minimize the ratio

BAG2 $=\frac{P_{2}}{P_{0}}$.

In order to check the performance of our two methods, we tested them using artificially generated, yet realistic, galaxies. In the first series of tests the artificial galaxies were simple axisymmetric exponential disks. We projected this disk using inclinations from $20^{\circ}$ to $80^{\circ}$. Then, these disks were interpolated with square grids with decreasing resolution, in order to simulate the effect of lower resolution in more distant galaxies. We investigated resolutions ranging from $300 \times 300$ pixels to $40 \times 40$ pixels. For all these cases, we applied our two methods and compared their results with the true values of the deprojection angles. In all the cases our methods performed extremely well. The deprojection angles were recovered exactly, except in the cases of very low resolutions and inclinations lower than $30^{\circ}$, where we obtained errors of the order of $\pm 2^{\circ}$ in the values of PA and IA.

We repeated these experiments adding a spiral component to the disk. The artificial galaxy is then described by

$$
\begin{aligned}
I(r, \theta)= & \mathrm{e}^{(-r / \alpha)} \\
& +A * \mathrm{e}^{\left(-r_{0} / \alpha\right)} \exp \left[-\left(\frac{r-r_{0}}{\sigma}\right)^{2}\right] \cos (p \ln (r)+2 \theta),
\end{aligned}
$$

where $r, \theta$ correspond to the polar coordinates and $\alpha, A, r_{0}, \sigma$ and $p$ are the constants of the model. In these cases we recovered again the deprojection angles with great precision, although not exactly. A typical example is given in Fig. 3. For inclinations lower than $30^{\circ}$ both methods recovered the PA with a precision of $\pm 4^{\circ}$. For inclinations higher than $30^{\circ}$ we recovered the PA with a precision of $\pm 2^{\circ}$. These error bars were doubled in the case of very low resolutions. In the case of the values of IA, all methods recovered the IA value exactly in all cases, except in the cases of very low resolutions where the precision of the values of IA was about $\pm 2^{\circ}$.

These tests show beyond doubt that both our methods perform very well, obtaining very accurate values of the deprojection angles for artificially generated, yet realistic, galaxies in a variety of situations. Our methods can perform very well for very inclined, nearly edge on galaxies, with inclinations as great as 80 degrees and they are also very reliable in the more difficult cases of galaxies nearly face on. An interesting result of the test is also the good performance of our methods in the case of very low resolutions. This indicates that our methods can be used also to obtain the values of the deprojection angles in the case of very distant galaxies. Thus, our methods can be applied with confidence to samples of galaxies of cosmological interest. We will apply these methods to the galaxies of the present sample and then compare the values of the deprojection angles determined by our methods with the angles determined by the rest of the methods that can be found in the literature.

\section{Deprojection of the galaxies}

We applied the two methods described in the previous section to the galaxies in the present sample. For each image we proceeded as follows. First we constructed a grid covering all the

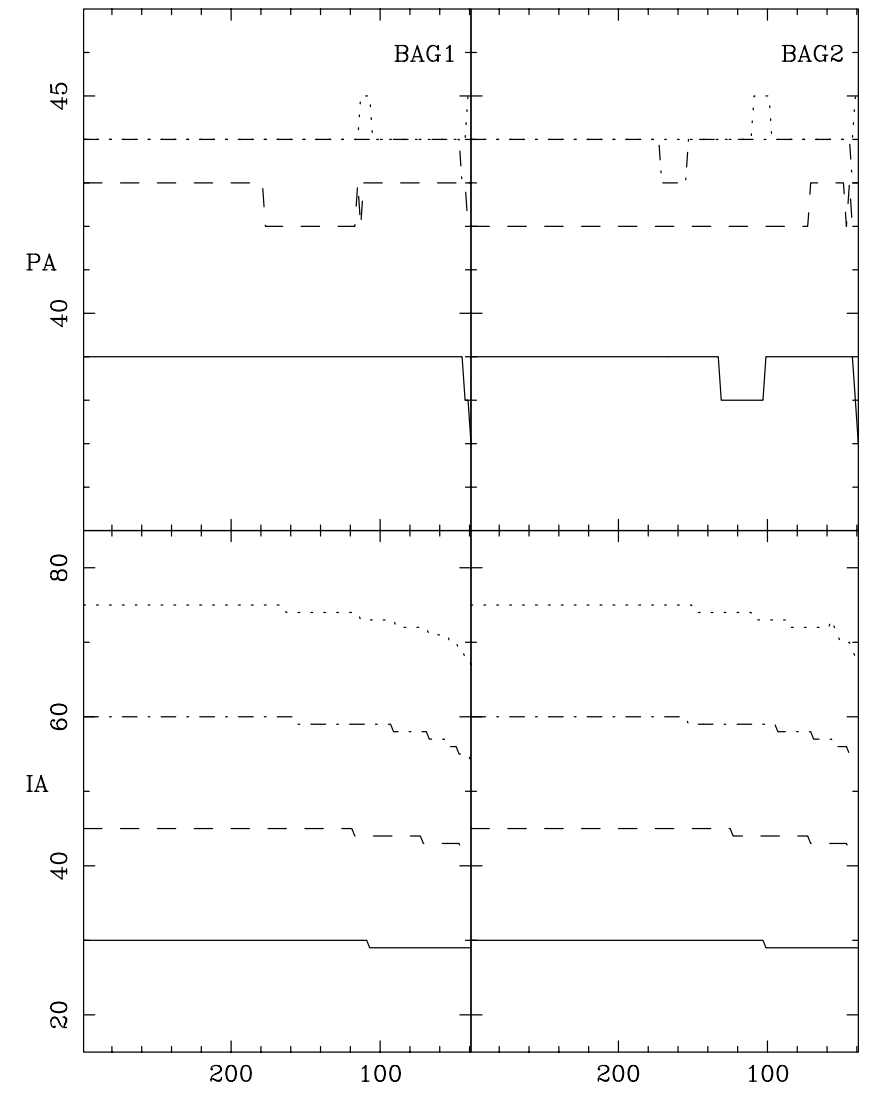

Fig. 3. Position (upper row) and inclination angle (lower row) found by our two methods. The results for BAG1 are in the left column, those for BAG2 in the right. The position angle is $45^{\circ}$ and the inclination angle $30^{\circ}$ (full line), $45^{\circ}$ (dashed line), $60^{\circ}$ (dot-dashed line) and $75^{\circ}$ (dotted line). Note the excellent performance of all methods in all cases. For this particular example we adopted $\alpha=30, A=0.1$, $r_{0}=25, \sigma=12.5$ and $p=2$.

possible range of values of the PA and IA in increments of 2 degrees. For each pair of angles (PA,IA) we deproject the galaxy image and we compute the Fourier transform (1) with the help of a polar grid. Using Eq. (2) we then calculate the power in each component and then the value of the ratios BAG1 and BAG2. We repeat this for every (PA,IA) pair on the grid. The optimum values are those for which we have a minimum.

We illustrate the use of our methods with the help of three galaxies: NGC 6503, NGC 5055 and NGC 3631. The first has been chosen as an example of a good case, the second of an intermediate and the third of a bad case. For each case, we give, on a rectangular grid of (PA,IA) values, two grey scale plots, one for the values of BAG1 and the other for the values of BAG2. Light shades denote low values and dark shades high values. We also superpose, on the grey scale plots, isocontours. The values in the parentheses above and to the right of each panel correspond to the values of the PA and IA that give the minimum value of the ratio in each case, i.e. the values that render the image most axisymmetric. The symbols depicted in each graph show the results found in the literature, obtained with other methods. For the ellipse fitting of the outer isophotes of an image we use the symbol $\mathrm{P}$, for values based on a kinematical analysis we use a $\mathrm{K}$ symbol followed by another letter 

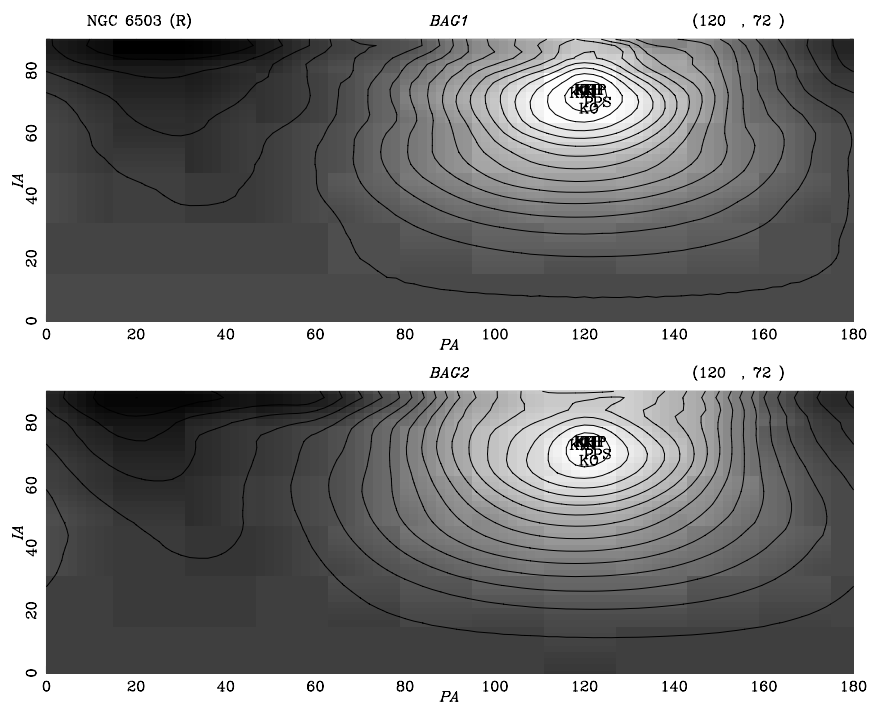

Fig. 4. Results of our methods for the galaxy NGC 6503 and the $R$ filter. The upper panel corresponds to our first method and the lower panel to the second method. We give the values of the PA and IA of the minimum in parenthesis in the upper right part of each panel. Symbols are described in the text.

to indicate the kind of data used. Thus KH indicates HI velocity fields, $\mathrm{KO}$ optical velocity fields, $\mathrm{KC}$ is used for $\mathrm{CO}$ velocity fields and KS for long slit measures. For methods based on the spiral structure we use a S. The " $\times$ " mark the position of the minimum values of BAG1 or BAG2, depending on the plot. The squares correspond to the mean weighted values given in Table 8 and obtained from BAG1 (or BAG2, respectively) and all the images of this galaxy and finally, the filled circle marks the location of the adopted values for this galaxy as explained in Sect. 6. Figure 4 shows our results for NGC 6503, a good case where the position of the minimum is well constrained by both methods, which further agree well between them and also with the rest of the estimates, as given by other methods found in the literature. In Fig. 5 we show the results for the galaxy NGC 5055.

NGC 3631 is an example of a very poor case and is shown in Fig. 6. As we can see, all the methods give values of the IA that are in general agreement, while the values of the PA are not well constrained. Only the kinematical methods are capable of constraining the value of this angle for galaxies with low inclinations. In Figs. 7-10 we show the images of NGC 3351, NGC 3486, NGC 4501 and NGC 5364, deprojected with the adopted deprojection angles.

For the computation of the Fourier transforms it is necessary to avoid the very central parts of the galaxies as we work with logarithmic radial coordinates $(u=\ln (r))$ and the center will give a singularity. In general it is sufficient to eliminate a relatively small part. For example, for the Frei sample we found it sufficient to eliminate the innermost part of the image, which is within a radius equal to $10 \%$ of its extent. There are two cases, however, where it is necessary to discard a larger region. The first case corresponds to galaxies with noticeable bulges. These, being nearly spherical, look round on the plane of the sky. When deprojecting the galaxy, the bulge will be stretched
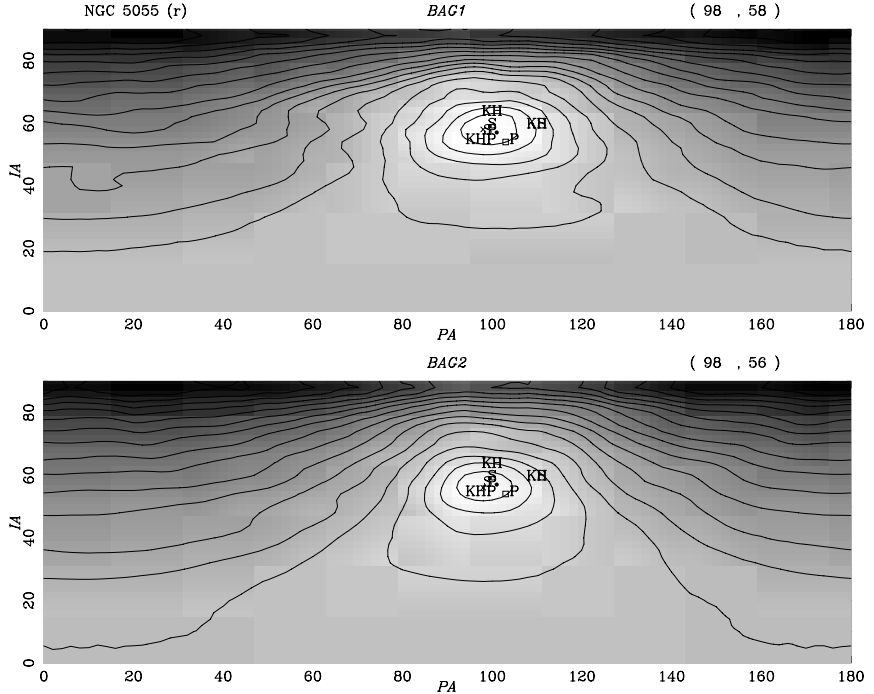

Fig. 5. Results of our methods for the galaxy NGC 5055 and the $r$ filter. Layout as in Fig. 4.
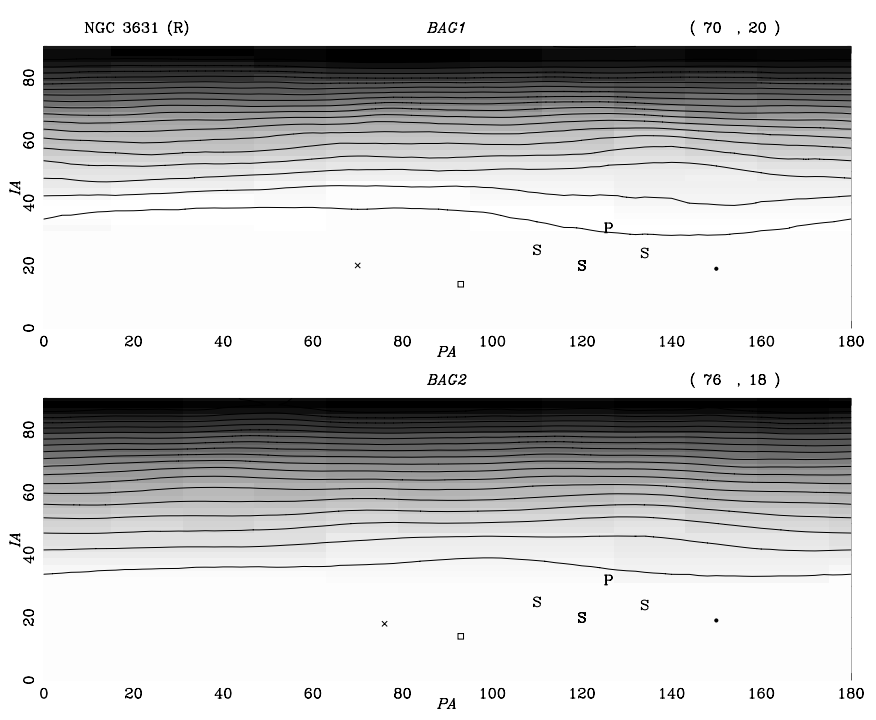

Fig. 6. Results of our two methods for the galaxy NGC 3631 in the $R$ band. Layout is as in Fig. 4.

to an elongated shape, thus giving strong spurious signals in the $m=2$ components that will bias results towards (PA,IA) $=$ $(0,0)$. Thus for this kind of galaxies it is necessary to avoid the bulge-dominated region and use mainly the disc in calculating the Fourier transform. For the Frei sample we found that, for such galaxies, we had to avoid the inner region within a radius equal to $20 \%$ of the extent of the image. For a few cases with great bulges like NGC 3031, it is necessary to go even to $30 \%$. Another galaxy type where is necessary to discard a more considerable region in the central parts of the image are the strongly barred galaxies, like NGC 3351 or NGC 4548. If we keep this inner structure, our methods try to render the bar circular and not the external disc. As the bars are normally of larger dimensions than the bulge, for these cases it is necessary to eliminate a region enclosing the whole bar and, in cases with an inner ring, also that ring. In some cases this includes a region within a radius equal to $50 \%$ of the extent of the image. In these 


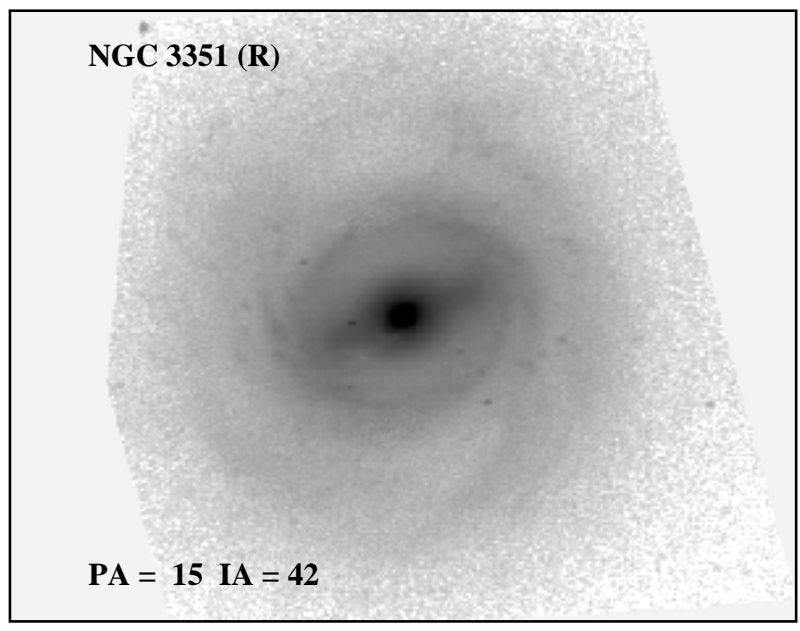

Fig. 7. Deprojected image of NGC 3351 in the $R$ filter.

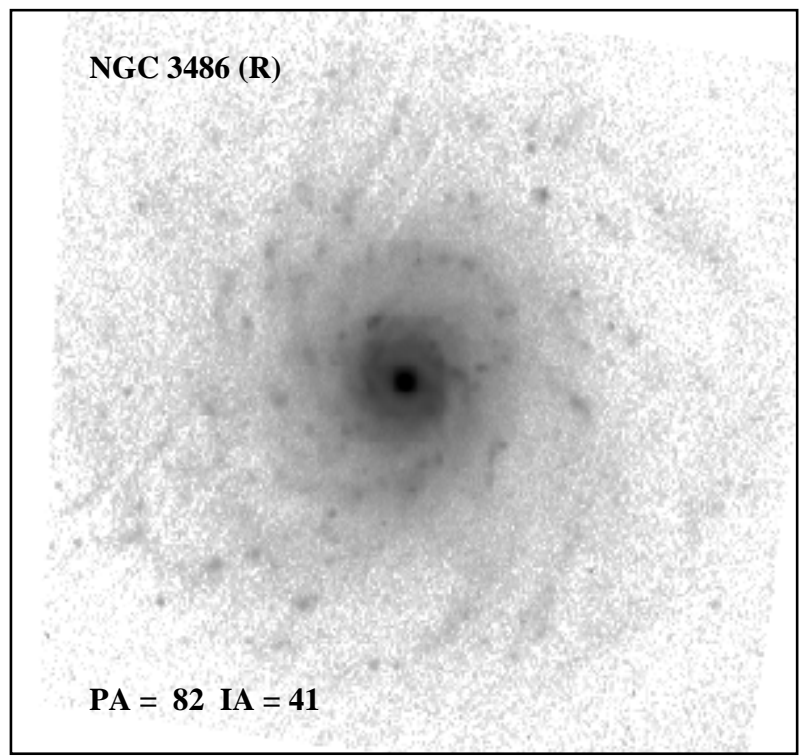

Fig. 8. Deprojected image of NGC 3486 in the $R$ filter.

cases, however, we are left with the less bright and noisier parts of the galaxy image and we can expect larger uncertainties.

It is crucial to consider how well our two methods constrain the deprojection angles they find. Indeed if the minimum in figures like 4,5 or 6 is very shallow, then any small perturbation, due to e.g. local structure on the images, may cause substantial changes in the values of PA and IA at which the minimum occurs, and thus large uncertainties $\triangle \mathrm{PA}$ and $\triangle \mathrm{IA}$. In order to quantify this effect we proceed as follows. We first find the minimum and maximum of all BAG1 and BAG2 values on the grid, and divide the range they delimit in 20 equal parts. We then calculate the number of pair values of PA and IA within the lower most $5 \%$ of this range and calculate the ratio, $N_{5}$, of the number of pair values in this bin over the total number of pair values. This value is a measure of the size of the well surrounding the minima. For well constrained values $N_{5}$ will be small and the larger it gets the greater is the uncertainty in the

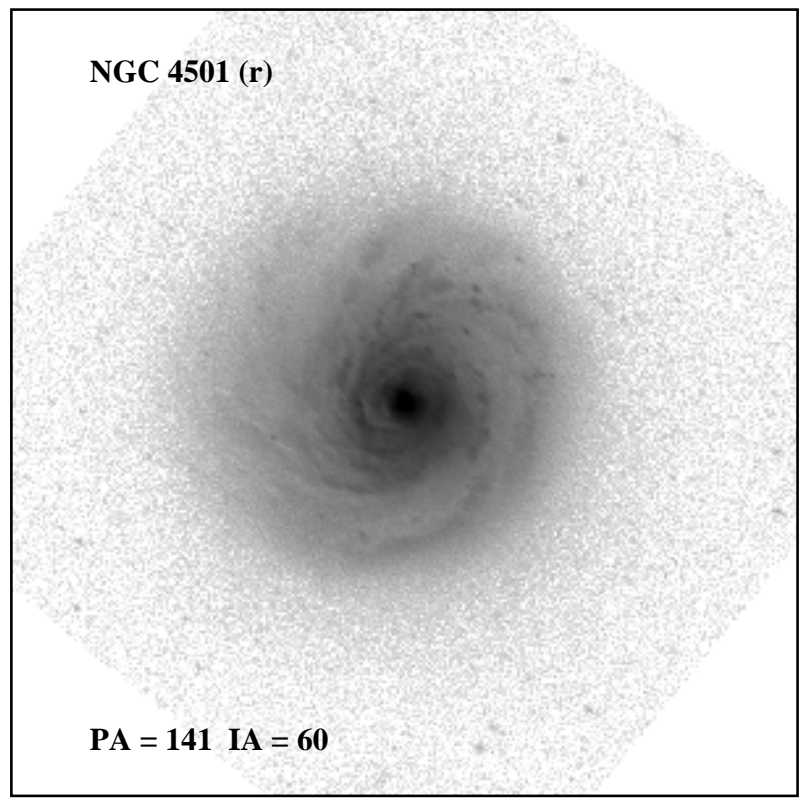

Fig. 9. Deprojected image of NGC 4501 in the $r$ filter.

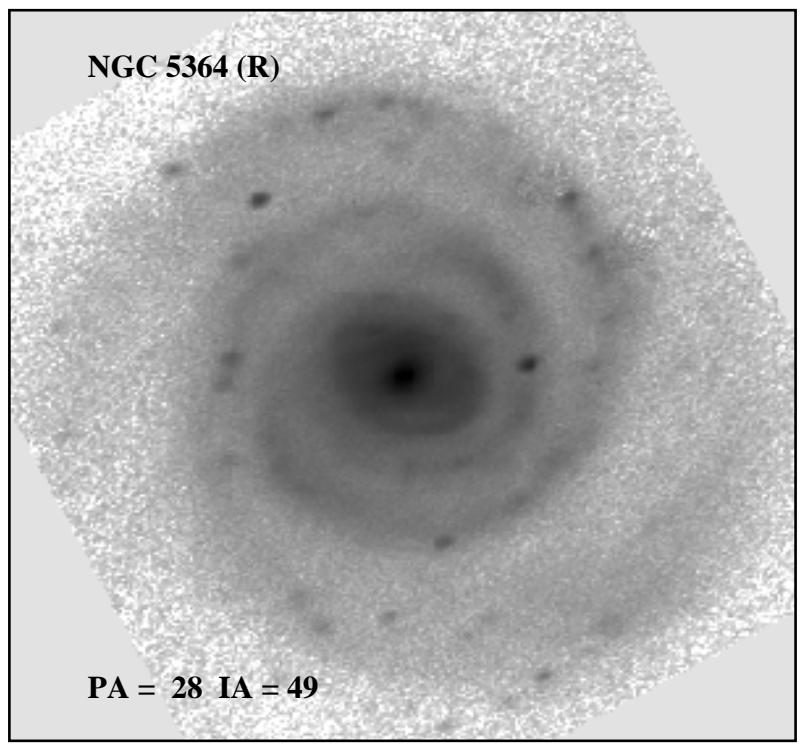

Fig. 10. Deprojected image of NGC 5364 in the $R$ filter.

determined values. We can thus use $N_{5}$ as a measure of how well constrained the values are.

Let us now compare the relative performance of our two methods. In Fig. 11 we plot the correlation between the $N_{5}$ values determined using the BAG1 method against the values determined by the BAG2 method. The solid line corresponds to the best fitting straight line with a correlation coefficient of 0.92 and a slope of 0.95 , while the dashed line indicates a one to one correlation. For this plot we use all the galaxies in the sample, combining the $r$ and $R$ pass-bands. It shows that the performance of the two methods is comparable, BAG2 giving somewhat better constrained values.

We next consider whether there is any dependency of the uncertainty on the color used to obtain the galaxy image. In Fig. 12 we give the correlations between the $N_{5}$ values 


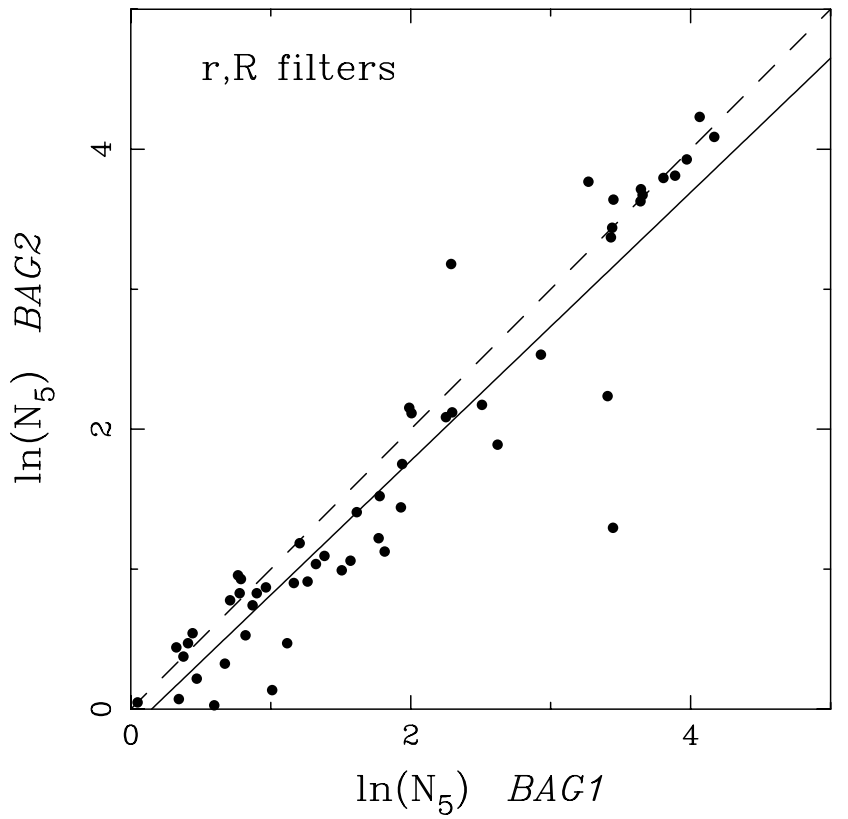

Fig. 11. Correlation between the $N_{5}$ values determined by applying our methods to the $r$ and $R$ pass-bands. The solid line corresponds to the best fitting line, while the dashed line indicates a one to one correlation.
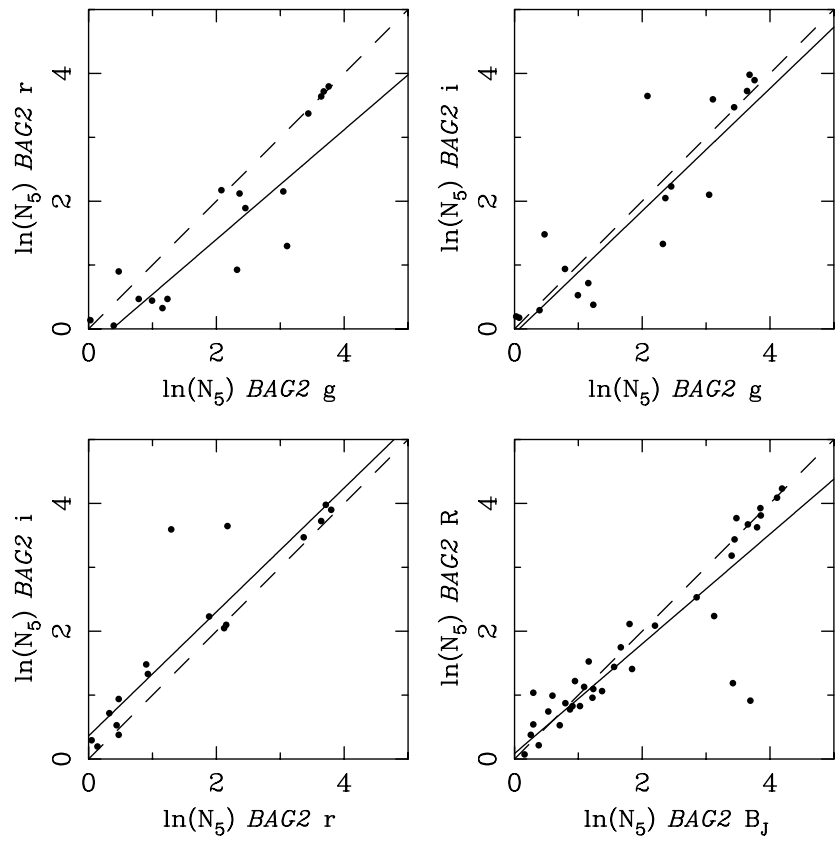

Fig. 12. Correlations for the $N_{5}$ values determined using the galaxy images in different pass-bands using the BAG2 method. The lines are as in Fig. 11.

obtained for the galaxy images in different pass-bands using the BAG2 method. It shows that there is a good correlation between the values obtained using different colors. The solid line indicates the best fitting straight line, while the dashed line is a one to one correlation. The values of the correlation coefficients range between 0.85 and 0.94 , the lowest values corresponding to the correlation between the $g$ and $r$ filters in the upper left panel. The slopes of the lines take values between 0.85 , for the

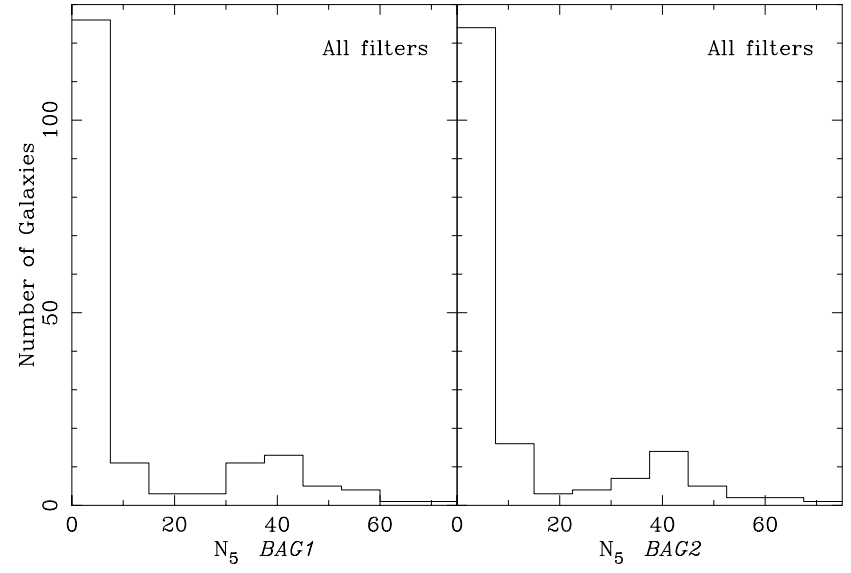

Fig. 13. Distribution of the number of galaxies as a function of the $N_{5}$ values for our two methods. The left panel corresponds to method BAG1, and the right panel to method BAG2.

$B_{J}-R$ correlation, and 0.97 , for the $r-i$ correlation. These plots show that the passband used in the galaxy image is not an important source of uncertainty in the determination of the deprojection angles. There is tendency for $R$ to fare better than $B_{J}$, and for $r$ to fare better than $i$, which in turn fares better than $g$, but the effect is small. The correlations for the values obtained using the BAG1 method are similar.

In Fig. 13 we show histograms of the distribution of the number of galaxies as a function of the $N_{5}$ value. The left panel corresponds to the values of the BAG1 method, while the right panel corresponds to the values of the BAG2 method. Based on the results of Fig. 12 we have pooled together in these plots all the images in the different pass-bands. As can be seen, for the great majority of images we obtain low values of the $N_{5}$ ratio, indicating that the deprojection values are well constrained. In both cases, however, the distribution seems to be bimodal, and there are a few galaxies for which we obtain large values of $N_{5}$, indicating that there are large uncertainties in these cases. Let us now consider the source of these large uncertainties.

The main source of uncertainty is in fact the orientation of the galaxy on the plane of the sky. For nearly face-on galaxies it is difficult to determine the IA and, particularly, the PA. Thus, for these galaxies we may expect greater uncertainties. This is shown in Fig. 14 where we plot the $N_{5}$ values as a function of the inclination angle, pooling together all the images in the $r$ and $R$ pass-bands. The lower panel corresponds to the BAG1 method and the upper panel to the BAG2 method. This figure shows that the deprojection angles are very well constrained by our methods for all galaxies with inclinations greater than 45 degrees. On the other hand, uncertainties may appear for galaxies with a lower value of the inclination angle. Such uncertainties are indeed expected for galaxies with inclination angles less than 35 degrees. Indeed, in such cases, methods based on images are not sensitive to the PA angle and one has to turn to kinematic methods. There are, however, galaxies with inclination angle between 35 and 55 degrees which have large uncertainties, and we want to examine the source of these uncertainties further. 


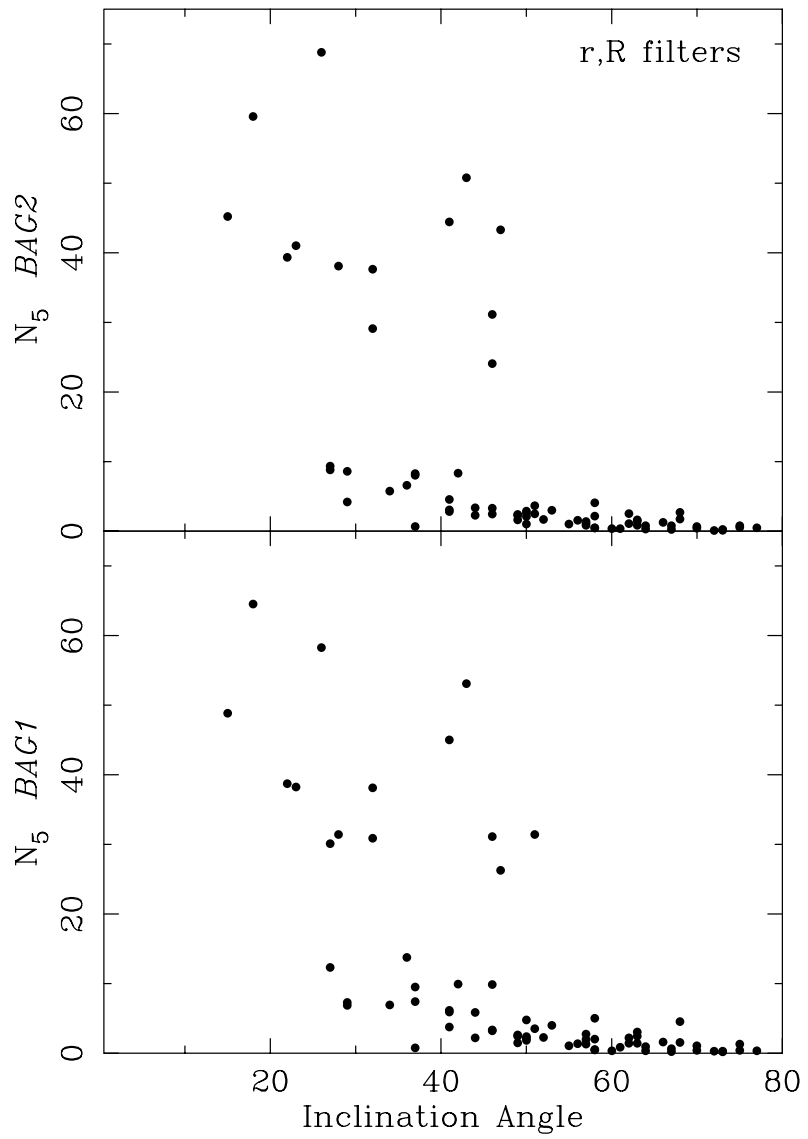

Fig. 14. Effect of the inclination on the uncertainties of the deprojection angles determined by our two methods. The lower panel corresponds to method BAG1 and the upper panel to method BAG2.

We find a systematic trend with family (i.e. with the existence of a bar). In Fig. 15 we plot $N_{5}$ as a function of bar strength using our two methods and restricting ourselves to galaxies with inclinations between 35 and 55 degrees. We note a systematic increase of $N_{5}$ with bar strength. Thus, the bar strength is a significant source of uncertainty for both our methods. This is due to the fact that for barred galaxies we use a smaller fraction of the image, thus making our methods less precise and less immune to "noise" or structure in the galaxy. To show this we took a non-barred galaxy, NGC 3486, and calculated $N_{5}$ discarding the innermost $50 \%$ of the image as for the case of strongly barred galaxies. We found a considerable increase of $N_{5}$, thus proving that it is the reduction of the fraction of the image that we use in the Fourier transform that introduces this uncertainty. Hubble type and Arm class could in principle also affect the uncertainties of our two methods. To test this we constrain ourselves to galaxies with inclination angles between 35 and 55 degrees and plot the values of the $N_{5}$ ratios as a function of Hubble type and Arm class. We did not find any significant trend, thus indicating that our methods are not affected by the Hubble type (i.e. the relative bulge size of the galaxy) or Arm class (i.e. the presence of strong arms). The former must be due to the fact that the central region carved out because of the bulge is smaller than that carved out

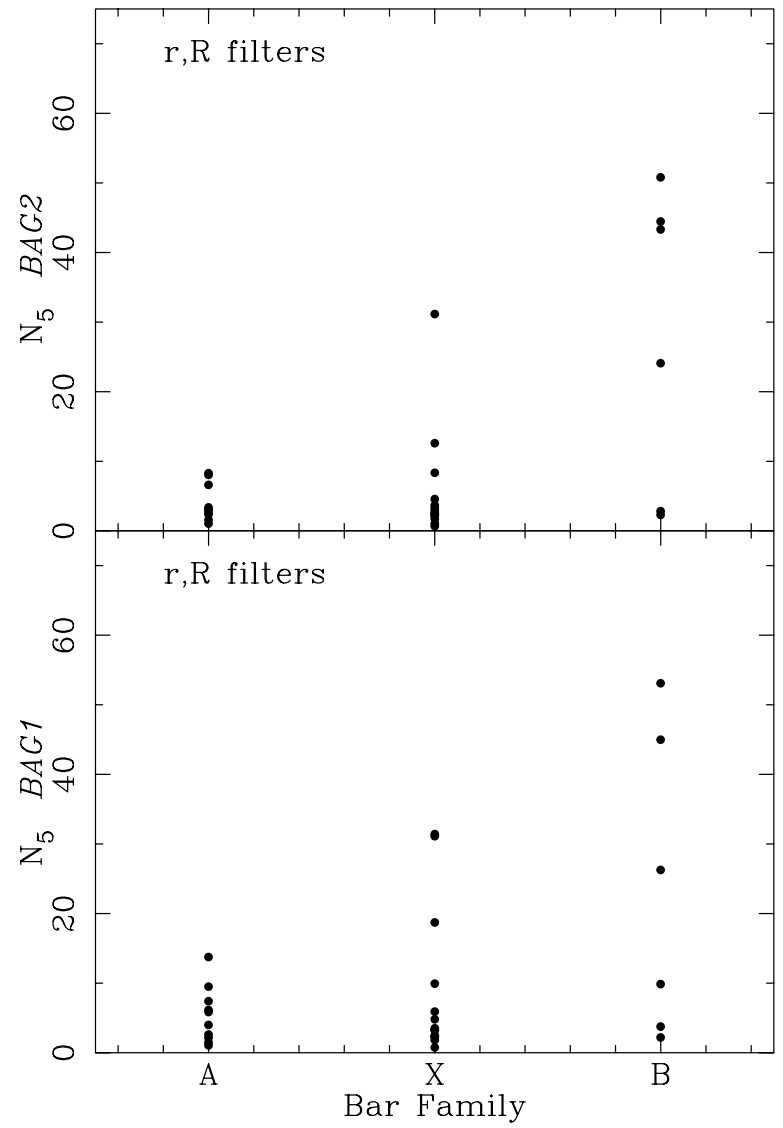

Fig. 15. Effect of the bar family type on the uncertainties of the deprojection angles determined by our two methods. The lower panel corresponds to the BAG1 method and the upper panel to the BAG2 method.

because of the bar, thus leaving sufficient disc area for the Fourier transforms.

\section{Comparison of the deprojection methods}

In this section we will compare the values of the deprojection angles determined by the different methods. In making the comparisons we grouped the methods in groups which are as homogeneous as possible. We thus group together all the values determined by the kinematics, as all authors use similar methods for data reduction. We also group together all values obtained by means of ellipse fitting to the outer isophotes. We use also the values of the RC3 (de Vaucouleurs et al. 1991) catalog as a single group, as they constitute a large and rather homogeneous sample. The same can be said for the values determined by Grøsbol (1985) and Danver (1942). We will also consider another group formed by all the previous determinations of the deprojection angles obtained from the different criteria on the power spectra obtained by bidimensional Fourier transforms on the HII region distribution of the galaxies. This gives us a total of six groups. 
For comparing any two methods we fitted a straight line to all pairs of values, using a maximum likelihood algorithm (Press et al. 1992), which minimizes the $\chi^{2}$ merit function

$\chi^{2}(a, b)=\sum_{i=1}^{N} \frac{\left(y_{i}-a-b x_{i}\right)^{2}}{\sigma_{y i}^{2}+b^{2} \sigma_{x i}^{2}}$,

where $\sigma_{x i}^{2}$ and $\sigma_{y i}^{2}$ are a measure of the errors for the $i$ th value. In the correlations including the PA, we assign weights according the IA, since galaxies with low inclination have illdefined position angles. These weights are taken from a linear function of the IA, such that this weight is zero for a face on galaxy $(\mathrm{IA}=0)$ and one for a galaxy with IA $=30$ degrees. For IA $\geq 30$ this weight is taken as unity. The $\sigma_{x i}$ and $\sigma_{y i}$ are taken as the inverse of the weights, i.e. $\sigma_{i}=1 / w_{i}$. For the correlations with the IA we use unweighted values, i.e. $\sigma_{x i}=\sigma_{y i}=1$.

Errors in the determination of the deprojection angles will introduce a scatter in the plot and lower the correlation coefficient as well as increase the mean of the orthogonal distances. We can use the parameters of these lines and the goodness of fit coefficients to measure quantitatively the relative performance of the different methods. For each straight line we obtain the value of the zero point $(a)$ and of the slope $(b)$, as well as two measures of the goodness of the correlation. These are the weighted mean of the perpendicular distances of the points to the straight line and the weighted correlation coefficient, defined as

$\rho=\frac{\sum_{i=1}^{N} w_{i}\left(x_{i}-\bar{x}\right)\left(y_{i}-\bar{y}\right)}{\sqrt{\left.\left.\sum_{i=1}^{N} w_{i}\left(x_{i}-\bar{x}\right)\right)^{2} \sum_{i=1}^{N} w_{i}\left(y_{i}-\bar{y}\right)\right)^{2}}}$,

where the means are defined as

$\bar{x}=\frac{\sum_{i=1}^{N} w_{i} x_{i}}{\sum_{i=1}^{N} w_{i}}, \quad \bar{y}=\frac{\sum_{i=1}^{N} w_{i} y_{i}}{\sum_{i=1}^{N} w_{i}}$.

In Figs. 16-18 we show examples of correlations between methods. In Fig. 16 we show the correlations between the values derived for the PA (left panel) and the IA (right panel) for our two methods. As we can see, there is an excellent agreement between them, with a weighted correlation coefficient of 0.97 for the PA values and 0.97 for the IA values. In Fig. 17 we show the correlations between our first method and the values derived from the kinematics. In this case, the correlation coefficients are 0.91 for the PA values and 0.86 for the IA values, showing again a good agreement between these two methods. Finally, in Fig. 18 we show the correlations between our second method and the values given in the RC3 catalogue (1991). The correlation coefficients are in this case 0.96 and 0.87 , again showing a good agreement. All the rest of the correlations have a similar shape and similar correlation coefficients.

The results of all the correlations are shown in Tables 2 to 6 . Table 2 shows the mean weighted orthogonal distances to the regression lines. The values above and to the right of the main diagonal correspond to the correlation of the PA values, while the values below and to the left of the main diagonal correspond to the correlations of the $\cos (\mathrm{IA})$ values. We use the $\cos (\mathrm{IA})$ instead of the IA values because the former are uniformly distributed in the sky. Table 3 shows the weighted
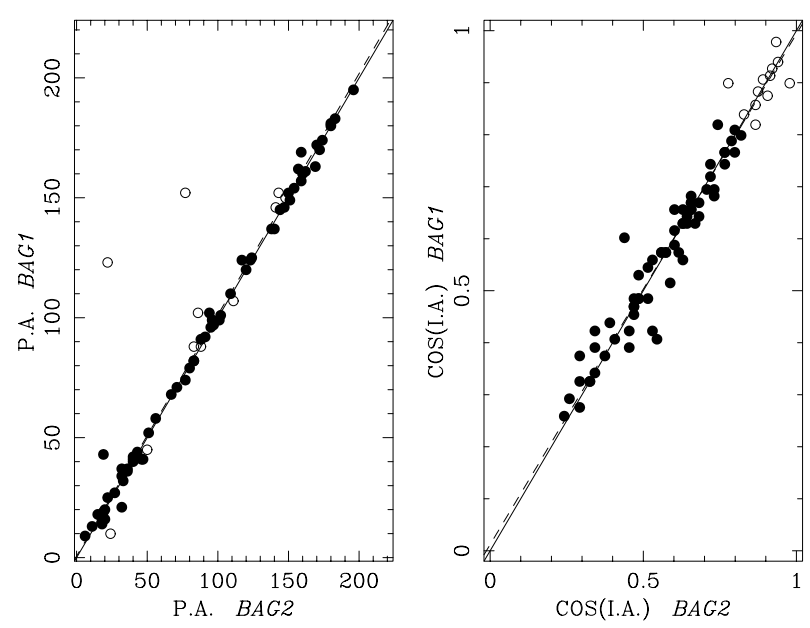

Fig. 16. Comparison off the PA values found by our two methods (left panel) and of the corresponding IA values (right panel). Galaxies with inclination less than 35 degrees are plotted with open symbols and the remaining ones with full symbols. The solid line gives the least squares fitting straight line, calculated as explained in the text, and the dashed one the diagonal.
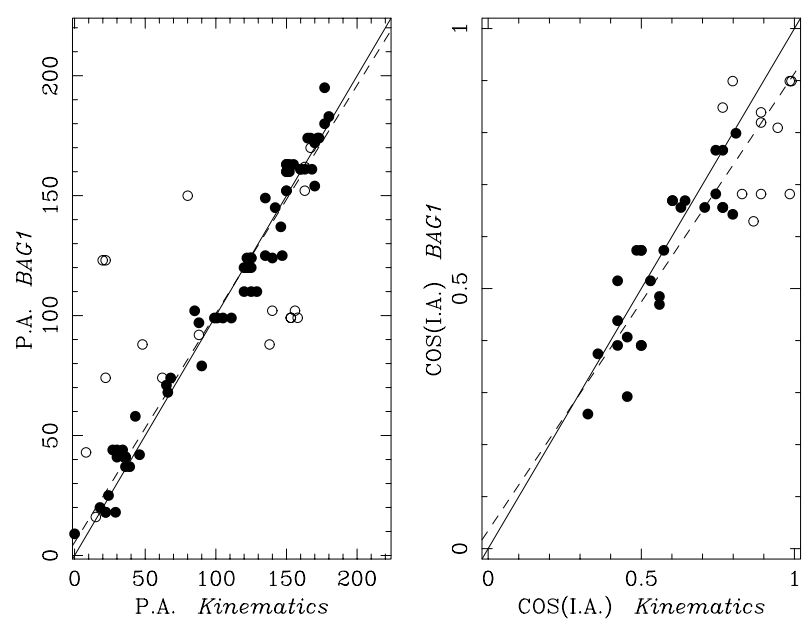

Fig. 17. Comparing the deprojected angles obtained with BAG1 with those obtained from the kinematics. The layout is as in Fig. 16.

correlation coefficients of the linear fits and Tables 4 and 5 the zero points and slopes of the straight lines respectively. Finally, Table 6 gives the number of points used in each correlation. Note that for a given pair of methods these numbers do not need to be equal for the PA and IA correlations, as some authors give only one of these values.

Both the figures and tables show that the PA values are better determined in a statistical sense than the IA values, in good agreement with what was already found by García-Gómez \& Athanassoula (1991) and García-Gómez et al. (2002). The mean correlation coefficient for the PA values is 0.86 , while the mean correlation coefficient for the IA is 0.81 . But this effect is only marginal.

We can not single out any particular method as being better than the rest, since all the correlations give both for the PA and IA zero points near zero and slopes near 1, thus indicating that none of the methods gives a systematic bias in the 
Table 2. Weighted mean orthogonal distances for the PA correlations (upper triangle) and the cos(IA) ones (lower triangle). All values are divided by their range interval and multiplied by 100 for the sake of comparison.

\begin{tabular}{rrrrrrrrr}
\hline \hline & BAG1 & BAG2 & RC3 & G & D & K & P & S \\
\hline BAG1 & & 1.79 & 3.53 & 5.68 & 5.24 & 5.10 & 8.24 & 6.10 \\
BAG2 & 2.06 & & 3.32 & 5.49 & 5.31 & 5.48 & 7.21 & 7.00 \\
RC3 & 4.68 & 4.45 & & 3.03 & 2.33 & 4.44 & 7.57 & 6.69 \\
G & 4.02 & 3.97 & 3.83 & & 5.15 & 6.92 & 10.16 & 6.90 \\
D & 4.71 & 4.55 & 4.05 & 5.43 & & 2.60 & 16.60 & 4.32 \\
K & 5.34 & 5.69 & 4.57 & 5.72 & 5.29 & & 9.41 & 2.89 \\
P & 4.68 & 5.13 & 6.14 & 5.59 & 6.43 & 7.95 & & 12.99 \\
S & 4.93 & 4.57 & 4.76 & 4.40 & 5.88 & 5.34 & 6.96 & \\
\hline
\end{tabular}

Table 3. Weighted correlation coefficients for the PA correlations (upper triangle) and the cos(IA) ones (lower triangle).

\begin{tabular}{rrrrrrrrr}
\hline \hline & BAG1 & BAG2 & RC3 & G & D & K & P & S \\
\hline BAG1 & & 0.97 & 0.95 & 0.91 & 0.84 & 0.91 & 0.74 & 0.88 \\
BAG2 & 0.97 & & 0.96 & 0.92 & 0.85 & 0.92 & 0.79 & 0.88 \\
RC3 & 0.87 & 0.90 & & 0.98 & 0.99 & 0.90 & 0.77 & 0.88 \\
G & 0.83 & 0.83 & 0.78 & & 0.93 & 0.84 & 0.72 & 0.86 \\
D & 0.89 & 0.91 & 0.90 & 0.76 & & 0.98 & 0.38 & 0.93 \\
K & 0.86 & 0.87 & 0.85 & 0.74 & 0.88 & & 0.70 & 0.98 \\
P & 0.78 & 0.78 & 0.74 & 0.55 & 0.69 & 0.55 & & 0.47 \\
S & 0.91 & 0.91 & 0.83 & 0.80 & 0.85 & 0.88 & 0.63 & \\
\hline
\end{tabular}
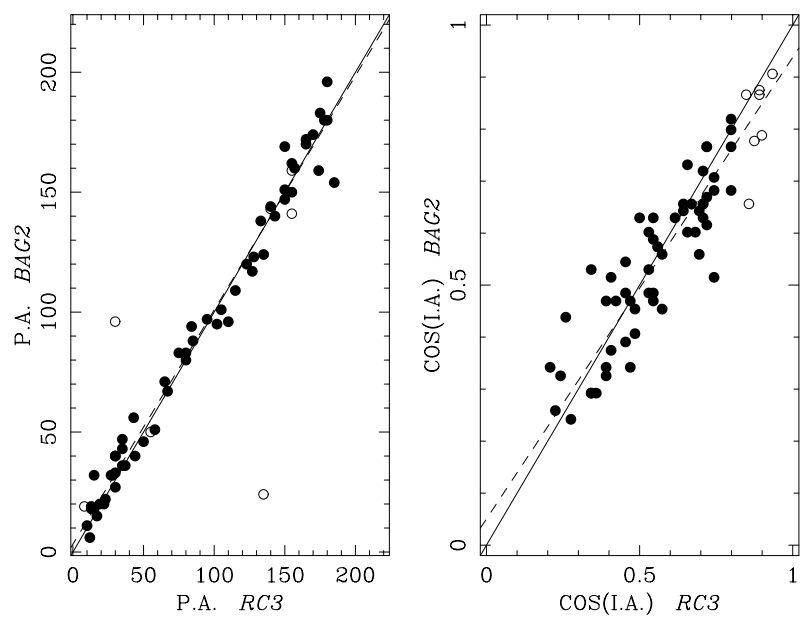

Fig. 18. Comparing the deprojected angles obtained with BAG2 with those obtained from the RC3 catalogue. The layout is as in Fig. 16.

determination of the values. We can also check the dispersion in each method either by computing the mean of the correlation coefficients of a method over the rest, or by using the mean distance. None of the methods has means which are strongly discrepant with the rest of the mean values. The lowest mean of the correlation coefficient and the highest mean distance is obtained for the values determined using the ellipse fitting to the outer isophotes, but this could be due to the fact that this is the most heterogeneous set of data. Our two methods give mean correlation coefficients with the rest of the methods of 0.89 for BAG1 and 0.9 for BAG2 in the case of PA and of 0.87 and 0.88 for the BAG1 and BAG2 method respectively in the case of IA. This indicates that our methods are well suited for the derivation of the deprojection angles. In general, we can conclude that all the methods for deriving the deprojection angles are well suited from a statistical point of view.

\section{Final adopted values}

For each galaxy we need to adopt a pair of deprojection angles that will be used for the subsequent analysis of the spiral structure and which is based in all the information obtained for that galaxy combining the results of different methods. The results of our analysis are shown in Table 7, which will be only published in electronic form at the CDS. In Col. 1 we give the galaxy name, then for each galaxy we give in Cols. 2-3 the results of method BAG1 for each of the filters. Columns 4 5 give the results of method BAG2. Column 6 give the filter used to obtain the image. Columns $7-8$ give respectively the PA and IA measured for this galaxy using other methods that were found in the literature. Column 9 gives a weight for these values. These were obtained deprojecting the galaxy image in the $r$ or $R$ pass-bands and are just a measure of the degree of roundness of this particular image using these particular values of the deprojection angles. Column 10 gives a code for the deprojection method used to obtain these values. For photometric techniques we use a $\mathrm{P}$, for kinematics we use a $\mathrm{K}$ symbol followed by another letter to indicate the kind of kinematics used. Thus, $\mathrm{KH}$ indicates $\mathrm{HI}$ velocity fields, $\mathrm{KO}$ optical velocity fields, $\mathrm{KC}$ is used for $\mathrm{CO}$ velocity fields and $\mathrm{KS}$ for long slit measures. For methods based on the spiral structure we use a S. In Col. 11 we give a code number to point to the reference from which these values were obtained, which is resolved at the end of the table. In Cols. 12-13 we give the mean PA and IA of the values obtained by method BAG1 using all the available 
Table 4. Zero points of the best fitting straight line for the PA correlations (upper triangle) and the cos(IA) ones (lower triangle).

\begin{tabular}{rrrrrrrrr}
\hline \hline & BAG1 & BAG2 & RC3 & G & D & K & P & S \\
\hline BAG1 & & 0.55 & 2.94 & 8.27 & -5.95 & 5.68 & 10.45 & -3.56 \\
BAG2 & 0.01 & & 3.26 & 5.42 & -2.82 & 3.59 & 7.89 & -8.37 \\
RC3 & 0.06 & 0.05 & & 1.48 & -4.43 & -8.10 & -2.82 & -22.80 \\
G & -0.05 & -0.02 & 0.05 & & -8.32 & -4.51 & 7.13 & -24.10 \\
D & 0.12 & 0.09 & 0.08 & 0.22 & & 4.16 & 21.44 & -0.99 \\
K & 0.03 & 0.03 & -0.02 & 0.25 & -0.04 & & 1.91 & -5.24 \\
P & 0.14 & 0.13 & 0.03 & 0.29 & -0.33 & -0.07 & & -17.18 \\
S & 0.02 & 0.04 & 0.05 & 0.17 & -0.06 & 0.01 & 0.12 & \\
\hline
\end{tabular}

Table 5. Slopes of the best fitting straight line for the PA correlations (upper triangle) and the cos(IA) ones (lower triangle).

\begin{tabular}{rrrrrrrrr}
\hline \hline & BAG1 & BAG2 & RC3 & G & D & K & P & S \\
\hline BAG1 & & 1.01 & 0.98 & 0.94 & 1.04 & 0.95 & 0.97 & 1.00 \\
BAG2 & 0.98 & & 0.98 & 0.94 & 1.02 & 0.95 & 1.00 & 1.00 \\
RC3 & 0.87 & 0.89 & & 1.01 & 1.03 & 1.02 & 1.08 & 1.11 \\
G & 1.07 & 1.03 & 0.98 & & 1.03 & 0.98 & 1.03 & 1.16 \\
D & 0.77 & 0.81 & 0.87 & 0.61 & & 0.97 & 0.94 & 1.00 \\
K & 0.88 & 0.89 & 1.03 & 0.61 & 1.06 & & 1.09 & 1.04 \\
P & 0.81 & 0.83 & 1.03 & 0.60 & 1.64 & 1.23 & & 1.03 \\
S & 0.96 & 0.94 & 0.92 & 0.72 & 1.18 & 0.99 & 0.76 & \\
\hline
\end{tabular}

filters for this galaxy. These mean values are the values that we will use for this galaxy and method BAG1. Column 14 gives a weight for these values as in Col. 9. Columns 15-17 give the same values as Cols. 12-14, but for method BAG2. Finally in Col. 18 we give the adopted PA value for this galaxy computed as the weighted mean of all the available values and in Col. 19 the uncertainty in this value of the PA, computed as the weighted dispersion. Columns 20-21 give the same values for the adopted IA. In Table 3 we give only the adopted values and their uncertainties for each galaxy. In Col. 1 we give the galaxy name, in Cols. 2-3 the adopted PA and its uncertainty $\triangle \mathrm{PA}$ and in Cols. $4-5$ the adopted IA and its uncertainty $\triangle I A$.

\section{Summary}

In this paper we introduce two new methods to obtain the deprojection angles of disc galaxies. These are based on two dimensional Fourier transforms of galaxy images. We also introduce a way of assessing the accuracy of these estimates. The methods perform particularly well for galaxies with inclinations greater than 55 degrees. For less inclined galaxies, the major source of uncertainty is the bar strength. For nonbarred galaxies our methods perform well up to inclinations of 30 degrees. The tests of our methods show as well that they can be used for low resolution images, thus making them appropiate for samples of galaxies of cosmological interest. A statistical comparison with the values of the deprojection angles determined using other methods shows good agreement between the various methods, thus enhancing confidence in statistical results. When deriving the values for a particular galaxy, however, it is best to apply more than one method to obtain the values of the PA and IA, since different methods introduce different biases. It can thus be that for a particular galaxy two
Table 6. Number of galaxies for the PA correlations (upper triangle) and the $\cos (\mathrm{IA})$ ones (lower triangle).

\begin{tabular}{rrrrrrrrr}
\hline \hline & BAG1 & BAG2 & RC3 & G & D & K & P & S \\
\hline BAG1 & & 76 & 66 & 48 & 29 & 89 & 124 & 48 \\
BAG2 & 76 & & 65 & 47 & 28 & 88 & 123 & 47 \\
RC3 & 66 & 65 & & 38 & 25 & 76 & 117 & 36 \\
G & 48 & 47 & 38 & & 18 & 46 & 94 & 30 \\
D & 29 & 28 & 25 & 18 & & 53 & 46 & 24 \\
K & 39 & 38 & 31 & 30 & 22 & & 107 & 145 \\
P & 122 & 121 & 115 & 92 & 46 & 73 & & 92 \\
S & 48 & 47 & 36 & 30 & 24 & 35 & 92 & \\
\hline
\end{tabular}

methods, because of their different biases, give rather different results, specially for the case of PA in nearly face-on galaxies. These biases do not make it possible to determine the deprojection parameters of a galaxy with a precision better than around five degrees. Finally, using a combination of the values obtained by our two methods and the rest of values found in the literature, we give a list of adopted deprojection angles with a measure of their respective uncertainty

\section{Appendix A: Deprojection software}

In order to facilitate the use of the two methods presented in this paper we make available upon request the software necessary for the PA and IA calculation. For those wishing to write their own software we give here some technical information on the methods.

We found it more straightforward to do the interpolation on the plane of the sky. For this we first define on the plane of the galaxy an appropriate polar grid, which we project on 
Table 8. Adopted values of the deprojection angles for the galaxies in the sample.

\begin{tabular}{|c|c|c|c|c|}
\hline Name & $\mathrm{PA}$ & $\Delta \mathrm{PA}$ & $\overline{\mathrm{IA}}$ & $\overline{\Delta \mathrm{IA}}$ \\
\hline NGC 2403 & 124 & 3 & 57 & 3 \\
\hline NGC 2541 & 170 & 2 & 57 & 7 \\
\hline NGC 2683 & 41 & 2 & 73 & 3 \\
\hline NGC 2715 & 19 & 3 & 68 & 4 \\
\hline NGC 2775 & 158 & 3 & 37 & 3 \\
\hline NGC 2903 & 20 & 4 & 61 & 3 \\
\hline NGC 2976 & 142 & 3 & 63 & 4 \\
\hline NGC 2985 & 178 & 6 & 37 & 3 \\
\hline NGC 3031 & 153 & 4 & 56 & 3 \\
\hline NGC 3147 & 150 & 5 & 32 & 4 \\
\hline NGC 3184 & 84 & 59 & 18 & 11 \\
\hline NGC 3198 & 39 & 3 & 70 & 3 \\
\hline NGC 3319 & 38 & 1 & 62 & 3 \\
\hline NGC 3344 & 156 & 1 & 24 & 6 \\
\hline NGC 3351 & 15 & 4 & 42 & 5 \\
\hline NGC 3368 & 157 & 6 & 50 & 1 \\
\hline NGC 3486 & 82 & 9 & 41 & 6 \\
\hline NGC 3596 & 155 & 8 & 27 & 2 \\
\hline NGC 3623 & 175 & - & 75 & - \\
\hline NGC 3631 & 152 & - & 20 & 6 \\
\hline NGC 3672 & 9 & 3 & 67 & 3 \\
\hline NGC 3675 & 0 & 1 & 66 & 1 \\
\hline NGC 3726 & 13 & 2 & 52 & 3 \\
\hline NGC 3810 & 25 & 6 & 44 & 2 \\
\hline NGC 3877 & 36 & 1 & 77 & 1 \\
\hline NGC 3893 & 171 & 5 & 50 & 1 \\
\hline NGC 3938 & 22 & 1 & 12 & 7 \\
\hline NGC 3953 & 14 & 2 & 58 & 4 \\
\hline NGC 4030 & 29 & 5 & 41 & 2 \\
\hline NGC 4088 & 52 & 6 & 68 & 2 \\
\hline NGC 4123 & 137 & 7 & 47 & 4 \\
\hline NGC 4136 & 92 & 3 & 29 & 8 \\
\hline NGC 4178 & 32 & 1 & 72 & 2 \\
\hline NGC 4189 & 81 & 9 & 44 & 5 \\
\hline NGC 4192 & 152 & 2 & 75 & 1 \\
\hline NGC 4216 & 19 & 1 & 73 & 5 \\
\hline NGC 4254 & 60 & 3 & 29 & 5 \\
\hline NGC 4258 & 160 & 4 & 64 & 3 \\
\hline NGC 4303 & 137 & 2 & 27 & 1 \\
\hline NGC 4321 & 153 & 4 & 28 & 3 \\
\hline NGC 4340 & 98 & 2 & 46 & 4 \\
\hline NGC 4394 & 111 & 5 & 23 & 2 \\
\hline NGC 4414 & 161 & 3 & 55 & 3 \\
\hline NGC 4450 & 174 & 5 & 50 & 3 \\
\hline NGC 4487 & 77 & 7 & 51 & 3 \\
\hline NGC 4498 & 136 & 2 & 58 & 2 \\
\hline NGC 4501 & 141 & 2 & 60 & 2 \\
\hline NGC 4527 & 67 & 1 & 70 & 2 \\
\hline
\end{tabular}

the plane off the sky. We calculate the values on the nodes of this 2D grid, using four points Lagrange interpolation using the Cartesian grid of the fits file. For deprojection purposes and depending on the resolution of the galaxy images we used grids with $256 \times 256$ or $512 \times 512$ nodes.
Table 8. continued.

\begin{tabular}{lrrrr}
\hline \hline Name & PA & $\Delta$ PA & IA & $\Delta$ IA \\
\hline NGC 4535 & 5 & 7 & 46 & 3 \\
NGC 4548 & 146 & 4 & 41 & 2 \\
NGC 4559 & 147 & 3 & 67 & 1 \\
NGC 4569 & 23 & 1 & 63 & 2 \\
NGC 4571 & 44 & 4 & 32 & 3 \\
NGC 4579 & 94 & 3 & 37 & 1 \\
NGC 4593 & 104 & 3 & 44 & 1 \\
NGC 4651 & 78 & 4 & 49 & 2 \\
NGC 4654 & 122 & 3 & 57 & 4 \\
NGC 4689 & 163 & 3 & 35 & 3 \\
NGC 4725 & 38 & 5 & 51 & 3 \\
NGC 4826 & 111 & 2 & 53 & 4 \\
NGC 5005 & 68 & 3 & 64 & 2 \\
NGC 5033 & 173 & 2 & 62 & 2 \\
NGC 5055 & 100 & 1 & 57 & 2 \\
NGC 5248 & 103 & 6 & 46 & 7 \\
NGC 5334 & 14 & 4 & 41 & 2 \\
NGC 5364 & 28 & 4 & 49 & 3 \\
NGC 5371 & 17 & 5 & 46 & 6 \\
NGC 5585 & 37 & 6 & 50 & 2 \\
NGC 5669 & 50 & 5 & 47 & 3 \\
NGC 5701 & 88 & - & 24 & 1 \\
NGC 5792 & 84 & - & 75 & - \\
NGC 5850 & 156 & 12 & 34 & 5 \\
NGC 5985 & 16 & 3 & 58 & 2 \\
NGC 6015 & 28 & 4 & 62 & 3 \\
NGC 6118 & 55 & 3 & 63 & 1 \\
NGC 6384 & 32 & 5 & 48 & 3 \\
NGC 6503 & 121 & 2 & 73 & 2 \\
\hline
\end{tabular}

Acknowledgements. We would like to thank A. Bosma for useful discussions. In preparing this paper we made extensive use of the CDS Strasbourg database. CGG and CB acknowledge financial support by the Dirección de Investigación científica y Técnica under contract AYA2001-0762. We also thank the Picasso program of bilateral exchanges under contract HF2001-023. This paper was finished while E.A. was in I.N.A.O.E. She thanks the I.N.A.O.E. staff for their kind hospitality and ECOS-Nord/ANUIES for a travel grant that made this trip possible.

\section{References}

Allsopp, N. J. 1979, MNRAS, 188, 765

Athanassoula, E., \& Misiriotis, A. 2002, MNRAS, 330, 35

Arsenault, R., Boulesteix, J., Georgelin, Y., \& Roy, J.-R. 1988, A\&A, 200, 29

Benedict, G. F. 1976, AJ, 81, 799

Begeman, K. G. 1987, Ph.D. Thesis

Blackman, C. P. 1979, MNRAS, 188, 93

Boselli, A., \& Gavazzi, G. 2002, A\&A, 386, 124

Boselli, A., Lequeux, J., Contursi, A., et al. 1997, A\&A, 324, 13

Braine, J., Combes, F., \& van Driel, W. 1993, A\&A, 280, 451

Brandt, J. C., Kalinowski, J. K., \& Roosen, R. G. 1972, ApJS, 24, 421

Braun, R., Walterbos, R. A. M., Kennicutt, R. C., \& Tacconi, L. J. 1994, ApJ, 420, 558

Bottema, R. 1988, A\&A, 197, 105

Boroson, T. A. 1981, ApJS, 46, 177

Boroson, T. A., Strom, K. M., \& Strom, S. E. 1983, ApJ, 274, 39 
Bosma, A., Goss, W. M., \& Allen, R. J. 1981, A\&A, 93, 106

Boggess, N. W. 1959, PASP, 71, 524

Burbidge, E. M., Burbidge, G. R., \& Prendergast, K. H. 1963, ApJ, 138,375

Buta, R. 1988, ApJS, 66, 233

Chincarini, G., \& Walker, M. F. 1967, ApJ, 149, 487

Comte, G., Monnet, G., \& Rosado, M. 1979, A\&A, 72, 73

Considère, S., \& Athanassoula, E. 1982, A\&A, 111, 82

Considère, S., \& Athanassoula, E. 1988, A\&AS, 76, 365

Corradi, R. L. M., Boulesteix, J., Bosma, A., et al. 1991, A\&A, 244, 27

Côté, S., Carignan, C., \& Sancisi, R. 1991, AJ, 102, 904

Danver, C. G. 1942, Lund. Obs. Ann., No. 10

de Jong, R. S., \& van der Kruit, P. C. 1994, A\&AS, 106, 451

de Vaucouleurs, G., \& Caulet, A. 1982, ApJS, 49, 51

de Vaucouleurs, G., de Vaucouleurs, A., Corwin, H. G., et al. 1991, Third Reference Catalogue of Bright Galaxies (New York: Springer) (RC3)

Elmegreen, D. M., \& Elmegreen, B. G. 1987, ApJ, 314, 3

Foster, P. A., \& Nelson, A. H. 1985, MNRAS, 215, 555

Fraternalli, F., van Moorsel, G., Sancisi, R., \& Oosterlo, T. 2002, AJ, 123,3124

Frei, Z., Guhathakurta, P., Gunn, J., \& Tyson, J. A. 1996, AJ, 111, 174

García-Gómez, C., \& Athanassoula, E. 1991, A\&AS, 89, 159

García-Gómez, C., Athanassoula, E., \& Barberá, C. 2002, A\&A, 389, 68

García-Ruiz, I., Sancisi, R., \& Kuijken, K. 2002, A\&A, 394, 769

Garrido, O., Marcelin, M., Amram, P., \& Boulesteix, J. 2002, A\&A, 387,821

Gottesman, S. T., \& Weliachew, L. 1975, ApJ, 195, 23

Grosbøl, P. J. 1985, A\&AS, 60, 261

Guhatakurta, P., van Gorkom, J. H., Kotany, C. G., \& Balkowski, C. 1988, AJ, 96, 851

Haynes, M. P., Giovanelli, R., Chamaraux, P., et al. 1999, AJ, 117, 2093

Héraudeau, Ph., \& Simien, F. 1996, A\&AS, 118, 111

Higdon, J. L., Buta, R. J., \& Purcell, G. B. 1998, AJ, 115, 80

Hunt, L. K., Malkan, M. A., Rush, B., et al. 1999, ApJSS, 125, 349

Irwin, J. A., \& Seaquist, E. R. 1991, ApJ, 371, 111

Iye, M., Okamura, S., Hamabe, M., \& Watanabe, M. 1982, ApJ, 256, 103

Jogee, S., Knapen, J. H., Laine, S., et al. 2002, ApJ, 570, L55

Kent, S. 1984, ApJS, 56, 105

Kent, S. 1986, AJ, 91, 130

Kent, S. 1987, AJ, 93, 81

Kent, S. 1988, AJ, 96, 514

Kornreich, D. A., Hayes, M. P., Lovelace, R. V. E., \& van Zee, L. 2000, AJ, 120, 139

Knapen, J. H. 1997, MNRAS, 286, 403

Knapen, J. H., Cepa, J., Beckman, J. E., del Rio, S., \& Pedlar, A. 1993, ApJ, 416, 563

Koopmann, R. A., Kenney, J. D. P., Young, J. 2001, ApJS, 135, 125

Laurikainen, E., \& Salo, H. 2000, A\&AS, 141, 103

Marcelin, M., Boulesteix, J., \& Georgelin, Y. 1983, A\&A, 1128, 140

Mathewson, D. S., \& Ford, V. L. 1996, ApJS, 107, 97

Meyssonier, N. 1984, A\&AS, 58, 351

Michard, R. 1985, A\&AS, 59, 205
Miller, B., \& Hodge, P. W. 1991, BAAS, 23, 966

Mölllenhoff, C., \& Heidt, J. 2001, A\&A, 368, 16

Moore, E. M., \& Gottesman, S. T. 1998, MNRAS, 294, 353

Moriondo, G., Giovanardi, C., \& Hunt, L. K. 1988, A\&AS, 130, 81

Münch, G. 1959, PASP, 71, 101

Oosterloo, T., \& Shostak, S. 1993, A\&AS, 99, 379

Phookun, B., Vogel, S. N., \& Mundy, L. G. 1993, ApJ, 418, 113

Press, W. H., Teukolsky, S. A., Vetterling, W. H., \& Flannery, B. P. 1992, Numerical Recipes (Cambridge Univ. Press)

Prieto, M., Longley, D. P. T., Perez, E., et al. 1992, A\&AS, 93, 557

Prieto, M., Gottesman, S. T., Aguerri, J. A. L., \& Varela, A. M. 1997, AJ, 114, 1413

Puerari, I., \& Dottori, H. A. 1992, A\&AS, 93, 469

Rhee, M.-H., \& van Albada, T. S. 1996, A\&AS, 115, 407

Rots, A. H. 1975, A\&A, 45, 43

Rots, A. H. 1980, A\&AS, 41, 189

Sakamoto, K. 1996, ApJ, 471, 173

Sánchez-Portal, M., Díaz, A. I., Terlevich, R., et al. 2000, MNRAS, 312,2

Schmitt, M., \& Kinney, A. L. 2000, ApJSS, 128, 479

Shostak, G. S., Willis, A. G., \& Crane, P. C. 1981, A\&A, 96, 393

Sicking, F. J. 1997, Ph.D. Thesis, Univ. Groningen

Sofue, Y., Tomita, A., Honma, M., \& Tutui, Y. 1999, PASJ, 51, 737

Stil, J. M., \& Israel, F. P. 2002, A\&A, 389, 42

Stocke, J. 1955, AJ, 60, 216

Swaters, R. A., \& Balcells, M. 2002, A\&A, 390, 863

Terndrup, D. M., Davies, R. L., Frogel, J. A., DePoy, D. L., \& Wells, L. A. 1994, ApJ, 432, 518

Thean, A. H. C., Mundell, C. G., Pedlar, A., \& Nicholson, R. A. 1997, MNRAS, 290, 15

Thornley, M. D., \& Mundy, L. G. 1997, ApJ, 484, 202

Thornley, M. D., \& Mundy, L. G. 1997, ApJ, 490, 682

Tully, B., Verheijen, M. A. W., Pierce, M. J., Huang, J. S., \& Wainscoat, R. J. 1996, AJ, 112, 247

Vallejo, O., Braine, J., \& Baudry, A. 2002, A\&A, 387, 429

van Albada, G. D. 1980, A\&A, 90, 123

van Albada, G. D., \& Shane, W. W. 1975, A\&A, 42, 433

van der Kruit, P. C. 1973, ApJ, 186, 807

van der Kruit, P. C. 1974, ApJ, 192, 1

van der Kruit, P. C., \& Shostak, G. S. 1982, A\&A, 105, 351

van Driel, W., \& Buta, R. J. 1991, A\&A, 245, 7

van Moorsel, G. A. 1983, A\&AS, 54, 1

van Moorsel, G. A., \& Wells, D. C. 1985, AJ, 90, 1038

Verdes-Montenegro, L., Bosma, A., \& Athanassoula, E. 1997, A\&A, 321,754

Verdes-Montenegro, L., Bosma, A., \& Athanassoula, E. 2000, A\&A, 356,827

Walterbros, R. A. M., Braun, R., \& Kennicutt, R. C. Jr. 1994, AJ, 107, 184

Weiner, B. J., Williams, T. B., van Gorkom, J. H., \& Sellwod, J. A. 2001, ApJ, 546, 916

Wevers, B. M. H. R., Appleton, P. N., Davies, R. D., \& Hart, L. 1984, A\&A, 140, 125

Wevers, B. M. H. R., van der Kruit, P. C., \& Allen, R. J. 1986, A\&AS, 66,505

Zasov, A. V., \& Sil'chencko, O. K. 1987, SvA Lett., 13, 186 